\title{
A Quality Improvement Initiative to Increase Colorectal Cancer (CRC) Screening: Collaboration between a Primary Care Clinic and Research Team
}

\author{
Green BB ${ }^{1,2 *}$, Fuller $\mathrm{S}^{1}$, Anderson $\mathrm{ML}^{1}$, Mahoney \\ $C^{1}$, Mendy $\mathbf{P}^{2}$ and Powell SL ${ }^{2}$ \\ ${ }^{1}$ Kaiser Permanente Washington Health Research \\ Institute, USA \\ ${ }^{2}$ Kaiser Permanente Washington, USA \\ *Corresponding author: Beverly B. Green, Kaiser \\ Permanente Washington Health Research Institute and \\ Kaiser Permanente Washington, 1730 Minor Avenue, \\ Seattle, WA 98101, USA
}

Received: March 07, 2017; Accepted: March 30, 2017; Published: April 05, 2017

\begin{abstract}
Background: Multiple randomized controlled trials have demonstrated that mailed fecal testing programs are effective in increasing colorectal cancer screening participation. However, few healthcare organization in the US have Implemented such programs.

Methods: Stakeholders from one clinic in an integrated healthcare system in Washington State initiated collaboration with researchers with expertise in $\mathrm{CRC}$ screening, aiming to increase screening rates at their clinic. Age-eligible individuals who were overdue for CRC screening and had previously completed a fecal test were randomized to receive mailed fecal immunochemical test kits (FIT) at the start of the project (Early) or 6 months later (Late). Outcomes included comparing FIT completion at 6 months by randomization group, and overall CRC screening rates at 12 months. We also assessed implementation facilitators and challenges.
\end{abstract}

Results: Overall 2,421 FIT tests were mailed at a cost of $\$ 10,739$. At 6 months, FIT completion was significantly higher among the Early compared to the Late group ( $62 \%$ vs. $47 \%, p<0.001)$. By 12 months, after both groups had received mailings, $71 \%$ in each group had completed a FIT. The clinic's overall CRC screening rate was $75.1 \%$ at baseline and $78.0 \% 12$ months later. Key constructs associated with successful program implementation included strong stakeholder involvement, use of evidence-based strategies, simplicity, and low cost. Challenges included lack of a plan for maintaining the program.

Discussion: Collaboration between clinic stakeholders and researchers led to a successful project that rapidly increased CRC screening rates. However, institutional normalization of the program would be required to maintain it.

Keywords: Quality Improvement; Healthcare system; Colorectal cancer screening; Primary Care

\section{Introduction}

Despite the potential of colorectal cancer (CRC) screening to reduce CRC mortality, CRC remains the second-leading cause of cancer deaths [1]. In 2017, an estimated 135,000 adults in the U.S. will be diagnosed with CRC, and 50,000 will die from the disease [1]. Better treatments have improved survival rates, but morbidity and mortality could be more rapidly and cost-effectively reduced by achieving higher uptake and adherence to CRC screening [2].

There are now multiple studies demonstrating that screening participation can be improved by mailing fecal tests directly to ageeligible individuals who are not current for CRC screening [3-8]. However, most health care organizations and clinics in the U.S. have not implemented mailed fecal test screening programs [3-8].

Group Health is a large, integrated health system with clinics in Washington State. One of the clinics in this system was interested in increasing its CRC screening rates. The clinic already had aboveaverage HEDIS (Healthcare Effectiveness Data and Information Set) CRC screening rate of over $74 \%$, compared to national average
HEDIS rate of $61 \%$ in 2015 [9]. However, the clinic wanted to do better, in part because one of their physicians died prematurely from CRC. Thus, two of the clinic stakeholders, a physician and clinical operations manager, contacted an expert in colorectal screening (Green) from Group Health Research Institute, who was conducting research in this area.

The group had several discussions and came up with some creative ideas to increase CRC screening, such as using videos in the waiting room, and sending out fecal test kits with a pamphlet telling the story of the physician who had died prematurely from CRC. His partner and daughter had given permission for his story to be used, in the hope that it would motivate patients to participate in CRC screening. The researchers provided input that the evidence of the effectiveness of narratives was scant, and that testing this idea would add to general knowledge but might or might not be successful in increasing screening rates. The researchers suggested an additional idea based on a sub-analysis of an ongoing trial, The Systems of Support to Increase CRC Screening and Follow-up trial (SOS, R01CA121125). In year 3 of the SOS trial, the research team re-randomized study participants
J Fam Med - Volume 4 Issue 3 - 2017

ISSN : 2380-0658 | www.austinpublishing group.com

Green et al. (C) All rights are reserved
Citation: Green BB, Fuller S, Anderson ML, Mahoney C, Mendy P and Powell SL. A Quality Improvement Initiative to Increase Colorectal Cancer (CRC) Screening: Collaboration between a Primary Care Clinic and Research Team. J Fam Med. 2017; 4(3): 1115 
who were still eligible for screening and who had received mailed fecal tests in years 1 and 2, to either Stopped or Continued mailed interventions. Compared to Stopped, Continued arm patients were significantly more likely to be screened at 12 months (net difference 16\%) [10]. However, the Continued mailed intervention was only successful in the subgroup of participants who had previously responded to mailed interventions during years 1 and 2 of the parent study. The net difference in screening rates between the Continued and Stopped groups was $2 \%, 11 \%$, and $27 \%$ among participants who had completed 0,1 , and 2 fecal tests in years 1 and 2 respectively. The percent of individuals completing fecal testing in the continued group was $18 \%, 45 \%$, and $77 \%$. This research suggested that mailing kits to patients overdue for screening, but who had previously completed fecal testing, might be an effective, efficient, and low cost way to increase screening rates

The clinic stakeholders and researchers discussed the evidence, examined the baseline data, made an evaluation plan, and determined the resources available to complete the project. This paper describes the collaboration between the clinic stakeholders and the researchers and the process for planning, implementation, evaluation, and maintenance of the project to increase the CRC screening rates.

\section{Methods}

The study was supported in part by the clinic and in part by a grant from the National Cancer Institute (R01CA121125). Group Health's institutional review board reviewed the project and determined that it constituted quality improvement, meaning that full IRB review was not required, and the need for patient consent was waived.

\section{Setting}

The study was conducted was conducted from October 2015 to November 2016 at Group Health Cooperative, and within one clinic within Group Health primary care medical center in Olympia Washington that cares for about 44,000 patients. The clinic is an accredited patient centered medical home (PCMH) and usual care includes strategies at clinic visits (in-reach) and outreach to increase CRC screening uptake [11]. As part of PCMH, medical assistants use the electronic health record (EHR) and embedded reports to see if patients are overdue for recommended screening tests, immunizations, and care for chronic conditions and pre-order or provide these services, including giving out fecal immunochemical test (FIT) kits, or reminding the provider to discuss other CRC screening options if the patient is overdue. The PCMH also includes outreach outside of clinic visits. Group Health sends its members an annual birthday letter that includes information on screening, immunizations, or care that is overdue. Medical assistants also reviews EHR lists of the provider patients overdue for preventive and chronic care services and calls patients to remind and assist them in completing needed care. In Olympia, medical assistants call patients to see if they want a FIT kit mailed to them, or mail it directly without a call if there are no other care needs requiring a telephone call. Group Health laboratory facilities also distribute FIT kits to patients who are due for CRC screening when patients present to the lab for other reasons. Batch-ordering for FIT testing is in place at Group Health, meaning that the primary care provider signs one set of annual orders for patients eligible for CRC screening. When the patient sends the FIT kit to the lab, the order is already in place and is activated. In
February of 2017, after the study was completed Group Health was acquired by Kaiser Permanente, and the clinic is now part of Kaiser Permanente Washington.

\section{Planning the quality improvement project}

The clinic stakeholders and research team met several times to discuss different options and examine baseline data, with the goal of increasing HEDIS rates for CRC screening at the clinic. They applied for internal funding to create a pamphlet and video based on the story of the physician who died prematurely from CRC, but were not selected for funding, with a concern being that the narrative might be relevant only to their specific clinic. The stakeholders were disappointed but not deterred, and a decision was made to pursue the researchers suggested project of mailing FIT kits to patients who were overdue for CRC screening but who had completed at least one fecal test in the past.

\section{Project costs}

Material resources, including FIT kits and pre-paid return envelopes, were provided by the clinic. Mailings were conducted by the research institute's survey program, which had experience with mailing kits for the SOS study. Mailing costs were paid for in part by the clinic and in part by the research team, who received permission to use funding remaining from the study for this implementation project. The total cost of the mailings was $\$ 10,739$, which included personnel time and costs associated with mailing the kits.

\section{Patients}

EHR and administrative data were used to identify eligible patients among all enrollees at the Olympia Medical clinic on September 30, 2015. A total of 5,520 patients were identified who were age-eligible (age 51-75) for CRC screening, had no HEDIS criteria exclusions (enrolled in the prior calendar year, no CRC, no total colectomy), and were due for screening (or would be due for screening by December $31,2016)$. Of these, $3412(61.8 \%)$ had completed a fecal test in the past (any time since health plan enrollment), and were eligible for the quality improvement initiative intervention.

\section{Intervention}

The mailed intervention was adapted from the SOS study which includes up to 3 mailings. In the SOS study the first mailing includes a pamphlet on CRC screening choices based on US Preventive Services Task Force and Group Health guidelines that states that they are due for CRC screening and will soon receive a FIT kit unless they request one of the other recommended tests (colonoscopy or flexible sigmoidoscopy). The second mailing includes the FIT kit, pictographic instructions and a letter emphasizing the importance of completing screening. If the FIT kit is not received and processed by the lab within 3 weeks, a reminder letter is mailed. The intervention mailing materials are available at the National Cancer Institute Research-Tested Intervention Programs (RTIPs) web site [12].

For the quality improvement initiative, the clinic generally followed the SOS model, but decided not to send the introduction letter because of limited resources. The letter sent with the kit was signed by the medical director of the clinic.

\section{Randomization}

The clinic wanted all eligible patients to receive the intervention, 
Table 1: Key Elements of the Collaboration between Health Care Stakeholders and Researchers for Implementation of Evidence Based Practices.

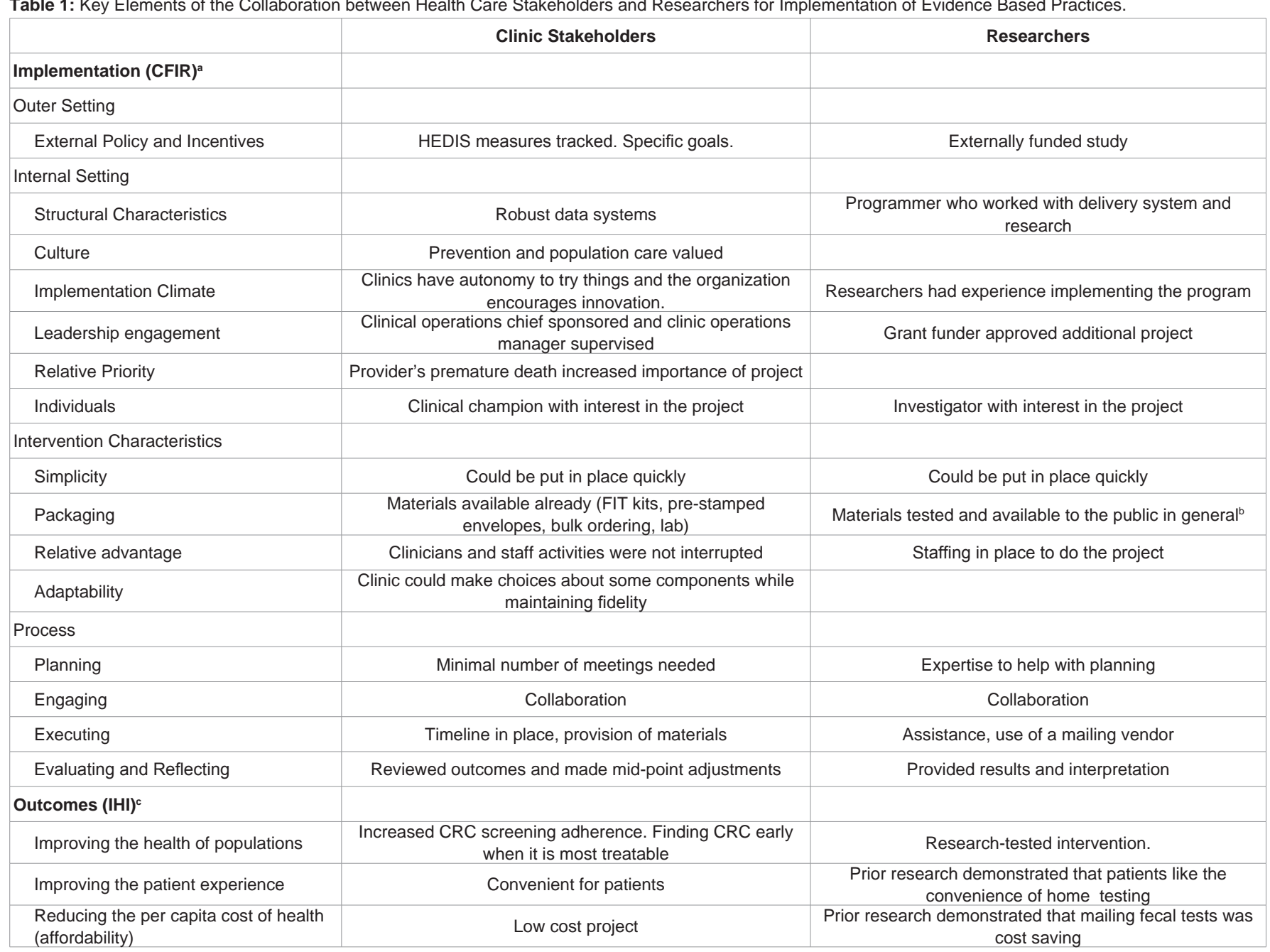

${ }^{a}$ CFIR: Consolidated Framework for Implementation Research http://www.cfirguide.org/constructs.html; representative constructs chosen

${ }^{\mathrm{b}}$ Research-Tested Intervention Programs (RTIPs)

https://rtips.cancer.gov/rtips/programDetails.do?programld=22691890

'The Institute for Healthcare Improvement Triple Aims.

http://www.ihi.org/engage/initiatives/tripleaim/pages/default.aspx

since the overall goal of the project was to improve clinic-wide CRC screening rates. To evaluate the success of the program, the researchers proposed that eligible patients be randomized to either Early or Late intervention groups, stratified by the numbers of fecal tests they had done prior ( 1 versus $>1)$. Patients in the Early group received intervention mailings within the first few weeks after randomization (mailings were completed between Oct 23 and Nov $20,2015)$, and patients in the Late group, who were still overdue for screening, received the intervention 6 months later (mailings May $2-6,2016)$. The effect of the mailed kit intervention was based on screening outcomes between randomization and 6months (before the Late group received the intervention). This also provided the clinic with an opportunity to stop the program if the expected increase in FIT uptake in the Early group did not occur.

\section{Evaluation}

The collaboration between the clinic and researchers provided an opportunity to explore key implementation and outcome factors, which the researchers used key domains from the Consolidated
Framework for Implementation Research (CFIR) and the Institute for Healthcare Improvement (IHI) "Triple Aims" to describe (Table 1) $[13,14]$. CFIR was developed by the Veterans Affairs Quality Enhancement Research Initiative as a framework for constructs that are associated with effective implementation of interventions or programs and takes into account contextual factors including the external environment (e.g. policies, incentives), internal setting (e.g. organization culture, priorities), individuals (e.g. champions, expertise), intervention characteristics (e.g. relative advantage, adaptability), and the process of change. The CFIR framework does not include outcomes, such as population reach, effectiveness, and costs, for which we used the IHI's Triple Aims, improving healthcare (excellent quality and service), health (improving patients' health outcomes and quality of life), and efficiency of care (providing efficient care and affordability).

\section{Analysis}

Our evaluation had two objectives: (1) to determine the incremental benefit of the mailed FIT program; and (2) to assess 


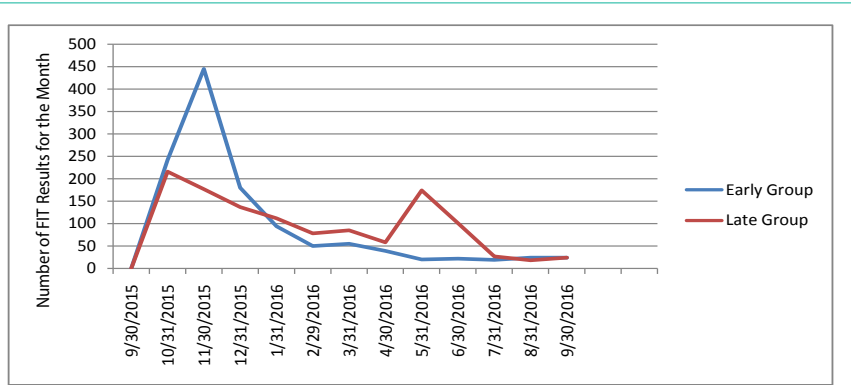

Figure 1a: Number of Fecal Immunochemical Tests (FIT) Completed between September 30, 2015 and September 30, 2016.

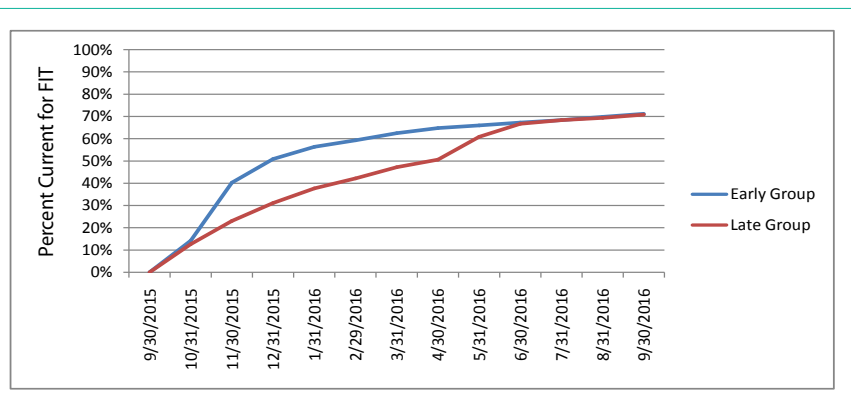

Figure 1b: Percent of Individuals Completing Fecal Immunochemical Testing in the Early and Late Group between September 30, 2015 and September 30, 2016.

the change in screening HEDIS rates. We assessed the incremental benefit of the mailed FIT kits by comparing Early and Late group FIT completion rates at 6 months (before the intervention was mailed to the Late group) to determine whether the mailed program resulted in an incremental increase in CRC screening over the usual practices the clinic implemented to increase screening uptake. We used chi-square tests to compare the proportion completing testing in each group. The total number of FIT kits completed and the cumulative proportion completing testing were plotted for each month by randomization group, to explore testing patterns over the study period. As the dates of the project did not match the calendar year, the impact of the initiative on clinic-wide screening rates was evaluated by comparing "HEDIS-like" rates. We call these measures "HEDIS-like", because we defined the evaluation periods as the 12 months prior and the 12 months following the randomization date rather than using calendar year, as the official HEDIS measure does. For each month following randomization, we computed a HEDIS-like rate, based on a rolling 12-month look back period.

\section{Results}

Overall 2,421 FIT tests were mailed, 1,706 kits to the Early group and 717 to the Late group 6 months later (the remaining 989 patients in the Late group were no longer due for CRC screening, either because they had completed screening, or were no longer eligible, e.g. disenrolled).

Figure 1 shows the number of FIT tests completed each month and the cumulative percent of each group that had completed testing during the 12 months after randomization. At 6 months, FIT test completion was significantly higher among the Early group $(n=1066 / 1706,62 \%)$ relative to the Late group $(n=805 / 1706$,
$47 \%)$, with a net difference of $15 \%(\mathrm{p}<0.001)$. An increase in FIT test completion was observed in the month following the mailing of kits to the Late group. However, the total number of completed tests following the second mailing was smaller than the Early group, since kits were not sent to Late group participants who had already completed screening, and those most likely to screen might have done so prior to the mailing. By the end of 12 months, there was no difference in the cumulative proportion which completed FIT testing between the 2 groups ( $71 \%$ in each group). Figure 2 shows change in the HEDIS-like measure over 12 months. The HEDIS-like rate was $75.1 \%$ at baseline prior to program startup and $78.0 \% 12$ months later (net gain of $2.9 \%$ ).

Key implementation and outcome factors and the interrelationship between the clinic, clinic stakeholders, and the study team are described in the Table 1 . Key constructs of the CFIR model were mostly satisfied, except for larger organizational investment in the project, as this work was conducted at one clinic rather than at all 26 of Group Health's primary care medical centers. The triple aim goal of improved healthcare, defined as improved CRC screening rates was also satisfied, and the program was low-cost.

\section{Discussion}

We describe here a clinic and researcher collaboration that led to increased CRC screening uptake. Particular strengths of this collaboration included the ability to share data and knowledge, shared-decision making on the pros and cons of different quality improvement strategies, and the ability to robustly evaluate the program.

Overall FIT testing rates among patients receiving the intervention were high at $71 \%$, but somewhat less than reports of studies or programs that focused on populations who had already completed fecal testing at least once. In the U.S., Baker et al. randomized primarily Hispanic Spanish-speaking community clinic patients who in the prior year completed mailed FIT screening, to receive either a mailed FIT kit program that included free kits,

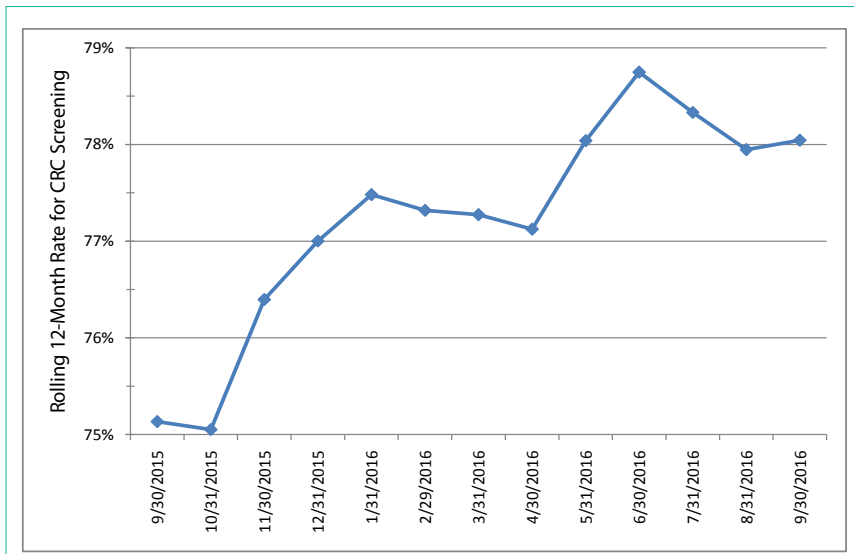

Figure 2: Change in Colorectal Cancer Screening rates from September 30 2015, to September 30, 2016, using HEDIS Specifications*.

*Denominator includes individuals ages 51-75 with at least two years enrollment, without prior colorectal cancer or colectomy. Numerator includes individuals from the denominator current for colorectal cancer screening defined as a fecal test in the prior year, flexible sigmoidoscopy in the prior 5 years, or a colonoscopy in the prior 10 years. 
low-literacy instructions, paid postage, automated text and phone reminders, and navigation; or usual care (stopped mailings) [6]. In the continued arm, over $82 \%$ completed FIT testing compared to $37 \%$ in the stopped arm. In our study, even though we only mailed FIT kits to patients who had done at least one fecal test prior, the completed test might have been done many years prior. In England, Lo et al. reported overall adherence rates of $66 \%$ in the $3^{\text {rd }}$ biennial round of a mailed fecal testing program [15]. However among individuals who had screened in both years 1 and 2 fecal test completion rates were $94.5 \%$ compared to $14.6 \%$ among individuals not screening in either year. Jenson et al. reported FIT testing rates of $75.3 \%$ to $86.1 \%$ in years 2-4 among individuals completing FIT testing in year 1 of the 4 year mailed FIT program at Kaiser Southern and Northern California [16]. However, in year 1 of the Kaiser program only $48.2 \%$ completed FIT testing, reaffirming that prior completion of FIT is a strong predictor of repetitive FIT adherence.

We demonstrated a $2.9 \%$ increase in HEDIS-like scores during the 12 months following randomization. The maximum increase was achieved at 9 months, after both groups had received mailings (78.7\%, net increase of 3.6\%). HEDIS rate increases would not be expected to be maintained unless the mailed interventions were implemented on an ongoing basis, as some people who were initially current for screening, would become due again as the year progressed. The team has discussed these issues and hopes that the overall organization will implement a centralized mailed kit program in the future. With improved screening rates during the initiative, we came close to, but did not reach the goal of the National Colorectal Cancer Round Table of $80 \%$ by 2018 [17]. We previously demonstrated that adding brief telephone assistance or nurse navigation increased the effectiveness of mailed fecal testing programs, particularly among individuals who had not previously participated in screening [4]. Adding these more intensive interventions would likely be necessary to attain higher screening completion targets.

Strengths of this project included collaboration between clinical staff and researchers, demonstrating the role each plays in implementing new programs. The clinics had strong opinion leaders and responsive quality improvement personnel. They persistently reached out to the research team after early attempts to get monies from the larger organization failed, and were able to contribute some of the resources needed to implement the project. The partnership helped us to design a project that could be robustly evaluated. This provides a model for other clinics and health organizations to adopt when attempting to change care practices.

Weaknesses include the lack of consent to use data on patient level characteristics such as sex, age, and ethnicity. We do not know whether the intervention worked well for specific subgroups, particularly for individuals with known CRC screening uptake disparities. Our project also did not address non-screeners, who might derive more benefit from starting CRC screening compared to those repeating testing. Lastly our initiative occurred within an organized health care system in which all patients had health insurance. Results might be different in community or safety net clinics.

While the program was successful in increasing screening rates, the clinic was not able to maintain the program, and the organization has not yet adopted it. The organizational business enterprise, rather than a single motivated clinic, would likely need to be engaged to ensure that the program could be sustained. However, small pilots such as this might be influential, and it remains possible that the organization will adopt a mailed program in the future.

\section{Conclusion}

Collaboration between clinic staff and researchers led to a successful project that rapidly increased CRC screening rates. While we succeeded in improving clinic-wide screening rates, institutional normalization of the program would be required to maintain the program.

\section{Funding Agency}

The National Cancer Institute of the National Institutes of Health (R01CA121125).

Registered at clinicaltrials.gov NCT00697047.

\section{References}

1. Siegel RR, Miller KD, Jemal A. Cancer statistics, 2017. CA Cancer J Clin. 2017; 66: 7-30.

2. Vogelaar I, van Ballegooijen M, Schrag D, Boer R, Winawer SJ, Habbema JD, et al. How much can current interventions reduce colorectal cancer mortality in the U.S.? Mortality projections for scenarios of risk-factor modification, screening, and treatment. Cancer. 2006; 107: 1624-1633.

3. Fielding J, Rimer B, Abraido-Lanza A, Calonge N, Clymer J, Glanz K, et al. Recommendations for Client- and Provider-Directed Interventions to Increase Breast, Cervical, and Colorectal Cancer Screening. Am J Prev Med. 2008; 35: S21-S25.

4. Green BB, Wang CY, Anderson ML, Chubak J, Meenan RT, Vernon SW, et al. An automated intervention with stepped increases in support to increase uptake of colorectal cancer screening: a randomized trial. Ann Intern Med. 2013; 158: 301-311.

5. Levy BT, Daly JM, Xu Y, Ely JW. Mailed fecal immunochemical tests plus educational materials to improve colon cancer screening rates in lowa Research Network (IRENE) practices. J Am Board Fam Med. 2012; 25: 7382.

6. Baker DW, Brown T, Buchanan DR, Weil J, Balsley K, Ranalli L, et al. Comparative effectiveness of a multifaceted intervention to improve adherence to annual colorectal cancer screening in community health centers: a randomized clinical trial. JAMA Intern Med. 2014; 174: 1235-1241.

7. Sequist TD, Zaslavsky AM, Marshall R, Fletcher RH, Ayanian JZ. Patient and physician reminders to promote colorectal cancer screening: a randomized controlled trial. Arch Intern Med. 2009; 169: 364-371.

8. Church TR, Yeazel MW, Jones RM, Kochevar LK, Watt GD, Mongin SJ, et al. A randomized trial of direct mailing of fecal occult blood tests to increase colorectal cancer screening. J Natl Cancer Inst. 2004; 96: 770-780.

9. Group Health Cooperative. 2015 HEDIS and CAHPS Measures and Performance. [cited December 29, 2016]. Available from: https://www.ghc. org/static/pdf/public/about/hedis.pdf.

10. Green BB, Anderson ML, Chubak J, Fuller S, Meenan RT, Vernon SW Impact of continued mailed fecal tests in the patient-centered medical home: Year 3 of the Systems of Support to Increase Colon Cancer Screening and Follow-Up randomized trial. Cancer. 2016; 122: 312-321.

11. Green BB, Anderson ML, Chubak J, Baldwin LM, Tuzzio L, Catz S, et al. Colorectal Cancer Screening Rates Increased after Exposure to the PatientCentered Medical Home (PCMH). J Am Board Fam Med. 2016; 29: 191-200.

12. National Cancer Institute. Smart Options for Screening (SOS). [cited January 30, 2017]. Available from: https://rtips.cancer.gov/rtips/programDetails. do?programld=22691890

13. Consolidated Framework for Implementation Research. CFIR Constructs. 
[cited November 20, 2016]. Available from: http://www.cfirguide.org/ constructs.html.

14. Institute for Healthcare Improvement. IHI Triple Aim Initiative. [cited November 20, 2016]. Available from: http://www.ihi.org/engage/initiatives/ tripleaim/pages/default.aspx.

15. Lo SH, Halloran S, Snowball J, Seaman H, Wardle J, von Wagner C Colorectcal cancer screening uptake over three biennial invitation rounds in the English bowel cancer screening programme. Gut. 2015; 64: 282-291.
16. Jensen CD, Corley DA, Quinn VP, Doubeni CA, Zauber AG, Lee JK, et al. Fecal Immunochemical Test Program Performance Over 4 Rounds of Annual Screening: A Retrospective Cohort Study. Ann Intern Med. 2016; 164: 456463.

17. Roundtable NCC. $80 \%$ by 2018. [cited November 20, 2016]. Available from: http://nccrt.org/tools/80-percent-by-2018/.
J Fam Med - Volume 4 Issue 3 - 2017

ISSN : 2380-0658 | www.austinpublishing group.com

Green et al. (C) All rights are reserved
Citation: Green BB, Fuller S, Anderson ML, Mahoney C, Mendy P and Powell SL. A Quality Improvement Initiative to Increase Colorectal Cancer (CRC) Screening: Collaboration between a Primary Care Clinic and Research Team. J Fam Med. 2017; 4(3): 1115 